\title{
EXPANSION IN SERIES OF NON-INVERTED FACTORIALS*
}

BY W. J. TRJITZINSKY

An expansion of the form

$$
f(z)=\sum \frac{b_{n}}{(z+1)(z+2) \cdots(z+n)}
$$

can be obtained from the consideration of Cauchy's formula

$$
2 \pi i f(z)=\int_{C} \frac{f(t) d t}{z-t},
$$

if $f(z)=0$ at infinity, together with the result $\dagger$

$$
\frac{n !}{(z+1)(z+2) \cdots(z+n+1)}=\int_{0}^{1} u^{n}(1-u)^{\star} d u,
$$

where $(1-u)^{z}$ denotes the branch reducing to unity for $u=0$. The above relations can also be used for deriving an expansion in series of non-inverted factorials. By (1) we have

Consider

$$
\frac{1}{z-t}=\int_{0}^{1}(1-u)^{z-t-1} d u \text {. }
$$

$$
(1-u)^{z-t-1}=(1-u)^{z}(1-u)^{-t-1} .
$$

Since

$$
(1-u)^{2}=1
$$

when $u=0$, we may write

$$
\text { (2) } \begin{aligned}
(1-u)^{z}=1-\frac{z}{1 !} u+\frac{z(z-1)}{2 !} u^{2}-\cdots \\
+\frac{(-1)^{n}}{n !} z(z-1) \cdots(z-n+1) u^{n}+\cdots
\end{aligned}
$$

* Presented to the Society, September 9, 1927.

$\dagger$ Whittaker and Watson, Modern Analysis, 3d edition, Cambridge University Press, 1920, p. 144. 
The binomial expansion (2) will be uniformly convergent for $0 \leqq u \leqq 1$ when $R(z)>0 .^{*}$ Also let us introduce the condition

that is,

$$
R(-t-1)>0
$$

$$
R(t)<-1
$$

Then the expansion

$$
\begin{aligned}
& (1-u)^{z-t-1}=(1-u)^{-t-1} \\
& +\sum_{n=1}^{\infty} \frac{(-1)^{n}}{n !} u^{n}(1-u)^{-t-1} z(z-1) \cdots(z-n+1),
\end{aligned}
$$

which holds for $0 \leqq u \leqq 1$, can be integrated termwise, so that we may write

$$
\begin{array}{r}
\frac{1}{z-t}=\int_{0}^{1}(1-u)^{z-t-1} d u=\int_{0}^{1}(1-u)^{-t-1} d u \\
+\sum_{n=1}^{\infty} \frac{(-1)^{n}}{n !} \cdot\left[\int_{0}^{1} u^{n}(1-u)^{-t-1} d u\right] \\
\cdot z(z-1) \cdots(z-n+1) .
\end{array}
$$

But

$$
\int_{0}^{1} u^{n}(1-u)^{-t-1} d u=\frac{-n !}{t(1-t)(2-t) \cdots(n-t)},
$$

and hence we have

$$
\text { (5) } \frac{1}{z-t}=-\frac{1}{t}-\sum_{n=1}^{\infty} \frac{(-1)^{n} z(z-1)(z-2) \cdots(z-n+1)}{t(1-t)(2-t) \cdots(n-t)} \text {, }
$$

where $R(z)>0, R(t)<-1$.

Let $R_{n}(u)$ denote the remainder after $(n+1)$ terms of the series (2) multiplied by $(1-u)^{-t-1}$. Since (2) is uniformly convergent, given $\epsilon, n_{0}$ can be found so that for $n \geqq n_{0}$ and all $u, 0 \leqq u \leqq 1$, we have

$$
\left|R_{n}(u)\right|<\epsilon .
$$

${ }^{*} R(z)$ denotes the real part of $z$. 
If we let $R_{n}^{\prime}(t)$ denote the remainder after $(n+1)$ terms of the series (5), we may observe that

and hence

$$
R_{n}^{\prime}(t)=\int_{0}^{1} R_{n}(u) d u
$$

$$
\left|R_{n}^{\prime}(t)\right| \leqq \int_{0}^{1}\left|R_{n}(u)\right| d u<\epsilon,
$$

where $\epsilon$ is independent of $t$; consequently, (5) is a uniformly convergent series in $t$.

Let $f(z)$ be a function analytic on and outside a closed contour $C$ situated to the left of $R(z)=-1$, and vanishing at infinity; then

$$
\begin{aligned}
-2 \pi i f(z) & =-\int_{C} \frac{f(t) d t}{z-t} \\
= & \int_{C} f(t)\left[\frac{1}{t}+\sum_{n=1}^{\infty}(-1)^{n} \frac{z(z-1) \cdots(z-n+1)}{t(1-t) \cdots(n-t)}\right] d t
\end{aligned}
$$

when $R(z)>0$. Since integration termwise is justifiable, we have

$$
\begin{aligned}
(6)- & 2 \pi i f(z)=\int_{C} \frac{f(t) d t}{t}+\sum_{n=1}^{\infty}(-1)^{n} \\
& \cdot\left(\int_{C} \frac{f(t) d t}{t(1-t) \cdots(n-t)}\right) z(z-1) \cdots(z-n+1) .
\end{aligned}
$$

Hence we may state the following theorem.

THEOREM I. Let $f(z)$ be a function analytic on and outside of a closed contour $C$ situated to the left of $R(z)=-1$, and vanishing at infinity; then for all $z$ with $R(z)>0$

$$
\begin{aligned}
f(z)=b_{0}+b_{1} z+ & b_{2} z(z-1)+\cdots \\
& +b_{n} z(z-1) \cdots(z-n+1)+\cdots,
\end{aligned}
$$

where

$$
b_{n}=\frac{(-1)^{n+1}}{2 \pi i} \int_{C} \frac{f(t) d t}{t(1-t) \cdots(n-t)}
$$


If we take $(1-u)^{z-t-1}$ as

$$
(1-u)^{z+k} \cdot(1-u)^{-t-1-k} \text {, }
$$

where $k$ may be complex, repeat the steps by means of which Theorem I was deduced, and replace $z$ by $z+k$ and $t$ by $t+k$, we find the following generalized theorem.

THEOREM II. Let $f(z)$ be a function analytic on and outside of a closed contour $C$ situated to the left of $R(z)=-R(1+k)$, and vanishing at infinity, then for all $z$ with $R(z)>-R(k)$, we have

$$
\begin{aligned}
& f(z)=b_{0}+b_{1}(z+k)+b_{2}(z+k)(z+k-1)+\cdots \\
& \quad+b_{n}(z+k)(z+k-1) \cdots(z+k-n+1)+\cdots,
\end{aligned}
$$

where

$$
{ }^{(10)} b_{n}=\frac{(-1)^{n+1}}{2 \pi i} \int_{C} \frac{f(t) d t}{(t+k)(1-t-k)(2-t-k) \cdots(n-t-k)} \text {. }
$$

Let $U$ be max. $|f(t)|$ on $C$ and $l$ the length of $C$; then considering the expansion defined by Theorem $I$, it is observed that $t=t_{1}+i t_{2}$ has $-t_{1}>1$, since $R(t)<-1$ so that $|n-t| \geqq n-t_{1}>n+1$, and hence

$$
\frac{1}{|t(1-t) \cdots(n-t)|}<\frac{1}{(n+1) !} \text {. }
$$

Consequently

$$
\left|b_{n}\right|<\frac{h}{(n+1) !} \quad\left(h=\frac{U l}{2 \pi}\right) .
$$

The University of Valparaiso 